\title{
PATOGENICIDAD Y SUSCEPTIBILIDAD A PHOMA EXIGUA Desm. DE TRES PORTAINJERTOS Y DOS VARIEDADES DE LIMONERO (CITRUS LEMON L.)
}

Besoain, X.A.; Gardiazabal, F. y Opazo, P.G. Facultad de Agronomía, Universidad Católica de Valparaiso. Casilla 4-D, Quillota, Chile.

Palabras clave: Phoma exigua, patogenicidad,atizonamiento, limoneros.

Key words: Phoma exigua, pathogenicity, shoot blight, lemons.

\section{RESUMEN}

En la localidad de La Serena, IVRegión del país, se detectaron plantas de limonero de dos años de edad con sintomas de atizonamiento a nivel de brotes $y$ presencia de cancros en el tronco con abundante exudación de goma, produciendo en algunos casos la muerte de las plantas. A partir de la zona de avance de estas lesiones se aisló en forma consistente micelio correspondiente a Phoma exigua Desm. A partir de aislamientos puros de este hongo se inocularon frutosy plantas de limonero, obteniéndose en estas últimas los mismos sintomas antes descritos. De este modo se demostró la patogenicidad de la cepa obtenida, la que fue recuperada a partir de las lesiones, manteniéndose la mismas caracteristicas del cultivo. Se realizaron además pruebas de susceptibilidad de dosvariedades de limón crecidas sobre tres portainjertos y la de éstos por separado. Los resultados demostraron que todas las combinaciones fueron afectadas, existiendo diferencias en susceptibilidad, siendo la variedad Génova más susceptible que la variedad Eureka.

Se concluye que esta sintomatologia, seria una nueva enfermedad que afecta al cultivo del limón, la que denominamos "Cancrosis y atizonamiento del limonero", causada por Phoma exigua Desm.

\section{INTRODUCCION}

A comienzos de la primavera de 1988 se detectó en un huerto de cítricos ubicado en la provincia de La Serena, localidad Pan de Azúcar, plantas de 2 años de edad de Citrus lemon L. var. Génova injertadas sobre Citrus macrophylla, las que presentaban lesiones necróticas o cancros y atizonamiento a nivel de ramas y troncos principales, un rápido colapso de ramas, y èn

\section{SUMMARY}

[Patogenicity and susceptibility of Phoma exigua Desm. to three rootstocks and two variety of lemon (Citrus lemon L).]

In La Serena, IV Region of Chile two years aged lemon plants (Citrus lemon L.), showing symtoms of shoot blight and cancrosis in the trunk together with plenty gum exudation were detected which, sometimes caused them to die. Considering progress of these lesions, micelium from the Phoma exigua Desm. species was isolated. Based on pure isolations of this fungus, fruit and lemon plants were inoculated which resulted in the same symthoms mentioned above. Thus pathogenicity of the resulting strain was proved, which was recovered from the lesions and maintained the same charactheristics of the culture. Besides two varieties of lemon plants grown in three rootstocks were submitted to proofs of susceptibility, while the latter were proved separately. Results showed that every combinations got affected revealing differences in susceptibility, being Geneve more liable than the Eureka variety. It is concluded that these symptoms mean anew disease that affecting lemon culture which we call "Cancrosis and shoot blight of lemon plant", caused by Phoma exigua Desm.

algunos casos la muerte de los árboles (Figura 1).

A partir de la zona de avance de las lesiones se detectó la presencia del género Phoma como causante de este problema. De acuerdo con antecedentes bibliográficos existe una especie del género Phoma afectando a cítricos, Phoma tracheiphilla, causante del "Mal secco", enfermedad que fue observada por primera vez en Sicilia en 1920 y fue atribuído después de muchos años de controversia al género Deuterophoma descrito por Petri en 1989 y reclasificado en el género Phoma en 1971 
(Ciccarone, 1971; Pionnat, 1982).

En relación a otras especies de Phoma no hay antecedentes que este patógeno afecte a plantas de cítricos, aunque sí existen ensayos relacionados con las pudriciones que causaria a distintas especies hortofrutícolas en post-cosecha (Paulson \& Schoeneweiss, 1971). Es así como en limones var. Génova se determinó a $P$. exigua como causante de una pudrición negra, firme, localizada en la parte basal de los frutos. Los daños se presentaban principalmente en la piel y en menor grado en la pulpa (Latorre, 1987).

Por otro lado, existen antecedentes de ataques de este patógeno a otros cultivos como Solanum tuberosum (раpa). Schoeneweiss (1972), describe a $P$. exigua como causante de atizonamientos severos en plantas ornamentales de Vinca minor, especialmente cuando existen condiciones de bajas temperaturas y fuertes lluvias. Los síntomas, en el caso de Vinca, aparecen inicialmente como lesiones a nivel del suelo, en los tallos, guías y en la base de brotes nuevos, provocando marchitez, oscurecimiento y muerte del tallo afectado. En el caso de Nerium se presenta un decaimiento y marchitez a nivel de brotes laterales.

Debido a que existen antecedentes previos de estos problemas patológicos asociados al género Phoma afectando a plantas de cítricos a nivel mundial ( Garnsey \& Timmer. 1988 ) y en atención a los síntomas y severidad del daño observado en terreno, se planteó la siguiente investigación que tuvo como objetivos:

- Determinar la patogenicidad del aislamiento correspondiente al género Phoma, aislado de limoneros enfermos.

- Identificar la especie del género Phoma aislada.

- Evaluar la susceptibilidad de este aislamiento en 2 variedades de limonero sobre 3 portainjertos, y sobre los portainjertos no injertados en relación a sus respectivos testigos.

\section{MATERIALES Y METODOS}

Para llevar a cabo esta investigación se realizaron aislamientos a partir de la zona de avance de lesiones presentes en plantas de limonero var. Eureka, provenientes de La Serena. aislándose en forma consistente en Agar papa dextrosa acidulado (APDA), micelio correspondiente al género P/roma. Para determinar la patogenicidad del hongo aislado se llevaron a cabo ensayos tanto en frutos de limón como en plantas, los que fueron realizados en la Estación Experimental La Palma, perteneciente a la Facultad de Agronomía de la Universidad Católica de Valparaíso, ubicada en la provincia de Quillota. VRegión.
Para este efecto se utilizó tanto el invernadero como el laboratorio de Fitopatología perteneciente a dicha Facultad.

Para la correcta identificación del aislamiento, se caracterizó tanto la colonia como las estructuras reproductivas del hongo, midiéndose 50 conidios y 50 picnidios. Para la correcta determinación de la especie, se enviaron 2 cultivo puros al Commonwalth Mycological Institute (CMI) Kew, Inglaterra.

\section{Patogenicidad en frutos.}

A partir del aislamiento de Phoma obtenido, se realizaron pruebas para determinar preliminarmente su patogenicidad en frutos de limón, inoculándolos con 2 trozos de $8 \mathrm{~mm}$ de diámetro de micelio puro del hongo cultivado en APDA, siendo depositados ambos trozos en lados opuestos en el fruto mediante una incisión con un sacabocado de $8 \mathrm{~mm}$ de diámetro. Los frutos fueron previamente desinfectados con hipoclorito de sodio al $1 \%$ por 5 minutos, posteriormente inoculados y colocados en cámara húmeda a $22^{\circ} \pm 3^{\circ} \mathrm{C}$ por espacio de 7 días. Se inocularon 4 frutos dejándose los respectivos testigos, a los que se les adicionó sólo APDA estéril.

Patogenicidad y evaluación de susceptibilidad en plantas de limonero.

Para la realización de las pruebas de patogenicidad se inocularon plantas de limonero de las variedades Génova y Eureka injertadas sobre: Citrus macrophylla, Citrus sinensis y Citrus volkameriana, inoculándose también los portainjertos sin injerto, y dejándose los respectivos testigos.

En el.caso de las plantas injertadas la variedad tenía un año de edad y los portainjertos 2 años. Las plantas provenían de un vivero comercial (donadas por Agrícola Huerto California, San Isidro, Quillota), las que se encontraban en contenedores de plástico negro, con un sustrato compuesto por arena, turba y tierra de hoja (proporción 1:1:1). El sustrato había sido previamente esterilizado con vapor a $80^{\circ} \mathrm{C}$ por 40 minutos.

La metodología de inoculación de las plantas fue la siguiente: en las plantas seleccionadas, se realizó un corte en bisel a $10 \mathrm{~cm}$ sobre la zona del injerto, adicionándose un trozo de micelio puro del hongo de $5 \mathrm{~mm}$ de diámetro, obtenido desde la zona de extensión a partir de un cultivo en APDA de 5 días de incubación, colocándose en contacto el micelio en la zona entre la corteza y el xilema. Una vez depositado el trozo de micelio, se envolvió la herida con una cinta plástica. Los testigos fueron tratados de igual forma, pero sólo con APDA estéril. En el caso de los portainjertos, éstos se inocularon mediante el mismo procedimiento que la variedad, procurando ser 
inoculados a una altura tal, que éstos tuvieran $1 \mathrm{~cm}$ de diámetro y dejándose los respectivos testigos.

Para evaluar las diferencias estadísticamente significativas a nivel de tratamientos se empleó un test de igualdad de medias.

\section{RESULTADOS}

Identificación de la especie del género Phoma aislada

En relación a las características del micelio del hongo aislado, éste presentaba en un principio un color gris-rosado, existiendo una leve tonalidad anaranjada, tornándose finalmente a un color negro. Este micelio se caracterizó por producir picnidios a los 20 días de edad. mantenido el cultivo bajo una condición de luz diurna.

Los picnidios eran de color café a negro, de forma globosa sin cuello visible y con un ostíolo simple, encontrándose insertos en el micelio. Estos picnidios presentaban un ancho promedio aproximado de $200 \mu \mathrm{m}$. En presencia de agua liberaban masas de conidios hialinos. unicelulares, de forma ovoide, las que median entre 3 a $4 \mu \mathrm{m}$ de ancho por 5.5 a $8.0 \mu \mathrm{m}$ de largo (Figura 2).

Las características anteriormente descritas más la identificación del aislamiento enviado al Commonwalth Mycological Institute (CMI), correspondieron a Phoma exigua Desm.

\section{Patogenicidad en frutos.}

De acuerdo con los resultados obtenidos se determinó la patogenicidad del aislamiento de Phoma exigua en frutos de limón. Este hongo se caracterizó por el desarrollo de una pudrición de consistencia firme de color café a negro existiendo necrosis tanto en la superficie como al interior del fruto.

A los 20 días post-inoculación los frutos se presentaban totalmente podridos o necrosados, cubiertos con micelio aéreo, observándoseademás una abundante formación de picnidios.

\section{Patogenicidad en plantas.}

En relación a las inoculaciones efectuadas en plantas de limonero con Phoma exigua, se comprobó su patogenicidad para todas las variedades y portainjertos señalados. causando un daño ascendente desde la zona de inoculación hacia el ápice. Este daño consistió inicialmente en un marchitamiento apical, existiendo luego formación de cancro en la zona inoculada y abundante exudación gomosa. para terminar con un atizonamiento o muerte del tejido. desde la zona inoculada hasta el ápice (Figura 3 y 4 ).
No obstante el resto de la planta, salvo unos centímetros desde la zona de inoculación hacia abajo, se encontraba aparentemente sana. De acuerdo con los tratamientos efectuados en las plantas inoculadas, se pudo recuperar la cepa de Phoma exigua.

Los síntomas obtenidos en este ensayo concuerdan con los observados a nivel de terreno en las plantas enfermas de la localidad de La Serena (Figura 1).

\section{Evaluación de la susceptibilidad varietal.}

Del ensayo de susceptibilidad llevado a cabo en las variedades Génova y Eureka, en combinación con los 3 portainjertos y de éstos sin injerto, se determinó que todos resultaron ser susceptibles a Phoma exigua, aunque presentaron diferentes grados de severidad de la enfermedad (Cuadro 1).

CUADRO 1. Susceptibilidad varietal evaluada en días de sobrevivencia para las diferentes combinaciones de plantas de limonero y de portainjertos, analizados mediante el test de igualdad de medias.

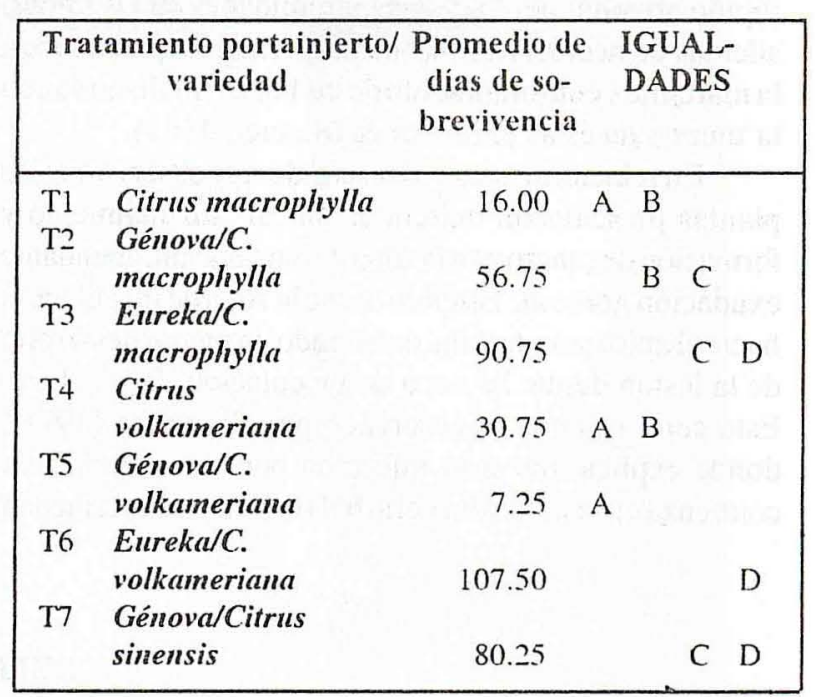

* Letras distintas indica diferencias significativas al $5 \%$.

Del Cuadro 1, se desprende que existe una diferencia en susceptibilidad entre variedades. siendo mayor en Génova y por otro lado. un efecto del portainjerto. situación que se visualiza al comparar el mayor grado de resistencia que posee la variedad Génova al estar injertada sobre $C$. marcophylla y $C$. sinensis en relación a la menor sobrevivencia estadisticamente significativa ( $\mathrm{P}=0.05$ ) sobre $C$. volkameriana. No obstante lo ocurrido en la variedad Génova, la combinación Eureka/C. volkameriana fue la que presentó el mayor grado de resistencia. sobreviviendo las plantas en promedio 107.5 dias. 


\section{DISCUSION}

De acuerdo con la revisión bibliográfica realizada, entre ellos el Compendio de Enfermedades de los Cítricos (1988), se desprende que no existen antecedentes de la especie $\mathbb{P}$. exigua afectando a plantas de limonero a nivel mundial. Por lo tanto, ésta sería la primera determinación de este taxon afectando a plantas de cítricos.

Existe una determinación previa de ataques de esta especie a los frutos de limón de la var. Génova en postcosecha (Latorre, 1987), los que concuerdan con los resultados obtenidos en la prueba de patogenicidad realizada a los frutos. No obstante, otra especie del género como $P$. tracheiphilla es causante de una importante enfermedad en plantas de cítricos denominada "Mal secco" (Bazzi \& Scrivani, 1954; Graniti, 1963; Tramier \& Mercier, 1963: Ciccarone,1971; Solel el al.,1972; Grasso, 1984).

Debido a la sintomatología que presentaban las plantas afectadas se podria haber pensado que los sintomas observados correspondían a los provocados por $P$. tracheiphilla, el cual provoca un desecamiento o rápido atizonamiento de las extremidades de las ramas, además de necrosis a nivel de la corteza, lo que induce a la marchitez y enrollamiento de las hojas, finalizando con la muerte de éstas (Tramier \& Mercier, 1963).

En relación a nuestros resultados, se observó que las plantas presentaban marchitez apical, atizonamiento y formación de cancroy en la zona de inoculación, abundante exudación gomosa. El tejido desde la zona de inoculación hacia el ápice se marchita. existiendo un menor desarrollo de la lesión desde la zona de inoculación hacia abajo. Esto contrasta con lo observado por Ciccarone (1971), donde explica que si la infección por $\boldsymbol{P}$. tracheiphilla comienza en la parte alta del árbol (brotes), la enfermedad se extiende a las hojas y las ramas principales y el colapso es mucho más lento, es decir, este patógeno puede infectar a la planta tanto basipétalmente como acropétalmente, situación levemente distinta a la observada en este trabajo.

Por otro lado, la combinación Génova/C. volkameriana fue una de las más susceptibles a la inoculación de $P$. exigua, dado que las plantas sobrevivieron en promedio 7,5 días. Estos resultados podrían compararse con los realizados por Grasso (1984), quien determinó grados de resistencia a $P$. tracheiphilla y ésta varía según la especie. Para Citrus lemon, escasa; $C$. macrophylla, escasa; $C$. volkameriana, media y $C$. sinensis elevada, pero no determinó un efecto con diferentes variedades.

Otro aspecto a considerar que diferencia a estas 2 enfermedades es lo expuesto por Ciccarone (1971) en donde, describe que las temperaturas óptimas para el desarrollo y producción de picnidios de $P$. tracheiphilla son entre los $20^{\circ} \mathrm{C}$ y $25^{\circ} \mathrm{C}$. Además, los conidios presentaban las siguientes características: hialinos. unicelulares, parcialmente curvados y con un tamaño promedio de $2,0-3,0 \mu \mathrm{m}$ por $1,0 \mu \mathrm{m}$, las que difieren significativamente de las medidas obtenidas del aislamiento de $\boldsymbol{P}$. exigua.

Al observar en el microscopio óptico las caracteristicas morfológicas del hongo aislado de plantas enfermas, éstas concuerdan con lo descrito por Boerema \& Howeler. (1967); Paulson \& Schoeneweiss (1971). para $P$. exigua. Por lo tanto, se concluye que esta sería una nueva enfermedad que afecta al cultivo del limón (Citrus lemon L.), la que de acuerdo con los síntomas observados podría ser denominada "Cancrosis y Atizonamiento del Limonero". causada por P. exigua Desm., existiendo una clara diferencia en la susceptibilidad a esta enfermedad de las 2 variedades evaluadas y el efecto que ejerce el portainjerto sobre ellas.

\section{REFERENCIAS}

Bazzi, B. \& Scrivani, P. (1954). Un método diagnóstico per il riconscimento del decorso del mal secco degli agrumi. Phytopathology Z. $21: 333-334$

Boerema, G. \& Howeler, L. (1967). Phoma exigua Desm. and its varietes. Persoonia . 5: 15-28

Ciccarone, A. (1971). Il fungo del mal secco degli agrumi. Phytopatologia Mediterranea $10: 68-75$

Garnsey, S.M \&Timmer, L.W. (Eds) (1988). Compendium of Citrus Diseases. APS . Florida.

Graniti, A. (1963). Picnidi di Deuterophoma tracheiphilla Petri. su organi fogliari. Phytopatologia Mediterranea 2:95

Grasso, S.( 1984). Il mal secco del limone. Informatore Fitopatológico $3: 39-42$

Latorre, B. (1987). Efecto de Phoma exigua en los citricos en postcosecha. Simiente $57: 253$
Paulson, G. \& Schoeneweiss, D. (1971). Epidemiology of Stem Blight of Vinca minor. Incited by Phoma exigua var. exigua. Phytopathology 61: 959- 963

Pionnat, J. (1982). Le mal sec Phoma tracheiphilla (Petri) Kone et Ghik: perspectives sur la lutte chimique et les varietes resistantes Fruits. 34: 237-248

Schoeneweiss, D. (1972). Control of stem blight of Vinca minor. Plant Disease Reporter 56: 238-241

Solel, Z.; Pinkias, J.; Loebenstein, G. (1972). Evaluation of sistemic fungicides and mineral oil adjuvants for the control of mal secco disease of lemon plants / Deuterophoma tracheiphilla. Phytopathology 62: 1007-1013

Traimer, R.\& Mercier, S. (1963). Sur la presence en France d' une maladi du citronnier, le mal sec, Deuterophoma tracheiphilla Petri. Rev: Path. Veg. et Ent. Agr. Fr. $62: 211-216$. 

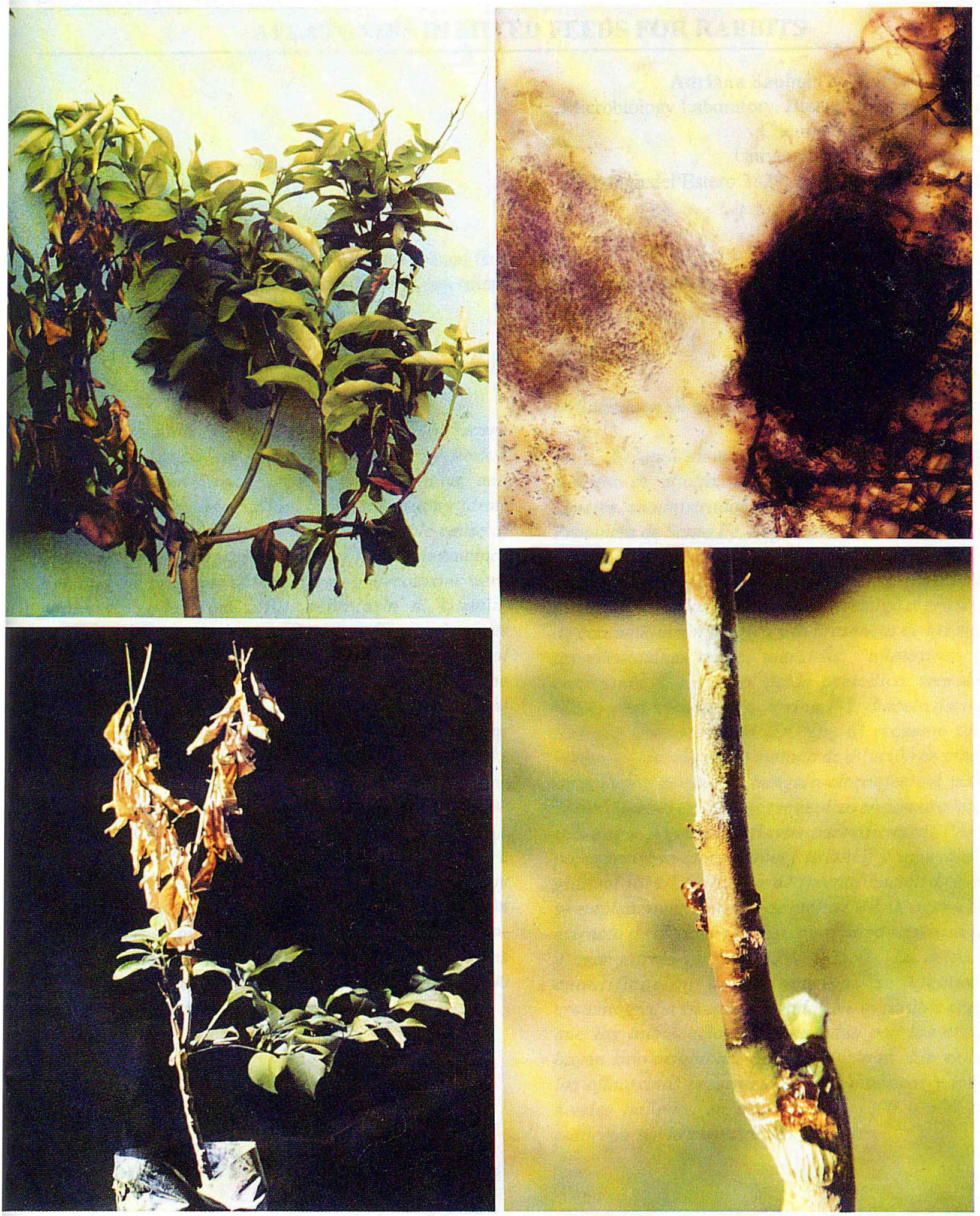

iguras 1, 2, 3 y 4: 1.- Planta de limonero var. Eureka provenientes de La Serena, con síntomas de atizanamiento ausado por Phoma exigua. 2.- Picnidio de Phoma exigua liberando conidios unicelulares por 200x 3.- Planta de monero var. Eureka injertada sobre C. macrophylla, inoculada con micelio de Phoma exigua, se aprecian los síntomas e atizonamiento del brote. 4.- Detalle de planta de limonero var. Eureka, se aprecia cancro a nivel del tallo con xhudación de goma. 